Dhaka Univ. J. Biol. Sci. 31(1): 79-91, 2022 (January) DOI: https://doi.org/10.3329/dujbs.v31i1.57918

\title{
TOXOPLASMA GONDII ENHANCES OXIDATIVE STRESS IN PREGNANT WOMEN: A CASE-CONTROL STUDY
}

\author{
Niladri Paul, Molie Rahman ${ }^{1}$, Atiqur Rahman and Taibur Rahman* \\ Laboratory of Infection Biology, Department of Biochemistry and Molecular Biology, \\ University of Dhaka. Dhaka-1000, Bangladesh
}

Key words: Toxoplasma gondii, Pregnant women, Seroprevalence, Oxidative stress, Antioxidant defences

\begin{abstract}
Toxoplasma gondii (T. gondii) is an intracellular protozoan parasite that causes the disease Toxoplasmosis. Acute infection of T. gondii in pregnant women is dangerous because the parasite can transmit into developing foetus and can cause congenital toxoplasmosis. To assess the prevalence of $T$. gondii infection, its association with pregnancy and the impact of this parasite on generating oxidative stress, ninety-two pregnant women and sixty-five age matched non-pregnant control subjects were enrolled in this study. Using an immunological assay with a rapid test cassette, $T$. gondii specific IgG antibody was detected in $25 \%$ pregnant women while it was found only $9.2 \%$ in non-pregnant healthy control subjects, suggesting that a higher percentage of pregnant women carried chronic T. gondii infection compared to control subjects. Anti-T. gondii IgM was also tested and found to be positive in only $3.3 \%$ pregnant women as compared to control. Polymerase Chain Reaction analysis was carried out with the DNA isolated from blood of both anti-T. gondii IgM and IgG positive pregnant women and was found to be negative for T. gondii REP-529 gene, suggesting a chronic infection. Our data suggest that pregnant women who were in contact with cat in their third trimester of pregnancy demonstrated a significantly higher association with acquiring infection. Furthermore, to determine any association of $T$. gondii infection with oxidative stress in pregnant women, oxidative stress markers were assessed in plasma samples. The total oxidative stress was found to be higher in T. gondii positive subjects compared to $T$. gondii negative samples whereas total antioxidant defence was observed to be slightly decreased in T. gondii positive pregnant women. It can primarily be said that chronic $T$. gondii infection in pregnant women may increase oxidative stress and this, therefore, may act as a risk factor for congenital toxoplasmosis.
\end{abstract}

\section{Introduction}

Toxoplasma gondii (T. gondii) is an intracellular protozoan parasite that causes a global endemic health problem known as Toxoplasmosis. It can infect all nucleated cells of warm-blooded mammals including humans ${ }^{(1,2)}$. There are two different types of Toxoplasmosis-Congenital and Acquired(2). Infection with the parasite T. gondii in human

*Author for correspondence: <taibur@du.ac.bd>. ${ }^{1}$ Bangladesh Institute of Research and Rehabilitation in Diabetes, Endocrine and Metabolic Disorders, Dhaka-1000, Bangladesh. 
is usually asymptomatic or with mild flu like symptoms. However, infection during pregnancy may cause foetal loss and congenital infection of the new-born ${ }^{(3,4)}$.Primary $T$. gondii infection acquiring throughout the gestation might lead to severe harm to the foetus, together with abortion,intrauterine growth retardation, stillbirth, early death or innate infection (5). In addition, T. gondii can cause severe effects on immunocompromised patients in diverse disease conditions, which include Acquired Immunodeficiency Syndrome (AIDS) ${ }^{(6)}$,Cancer ${ }^{(7)}$,organ transplantations ${ }^{(8)}$, diabetes mellitus due to chronic T. gondii infection mediated pancreatic beta cell destruction ${ }^{(9)}$, thalassemia ${ }^{(5)}$ etc.

Cat faecal matter is especially a dangerous route of transmission of the parasite ${ }^{(10)}$. Only 1 cyst consumed by a cat may end up in thousands of oocysts. These oocysts are resilient to harsh environmental conditions and may survive over a year in contaminated soil(11). It has also been reviewed that $16-39 \%$ of Bangladeshi population carries T. gondii antibodies in their blood serum(12). Importantly, it has been reported that $39 \%$ Bangladeshi pregnant women are infected with $T$. gondii ${ }^{(13)}$. Pregnant women are at more risk by this parasite because it causes abnormal pregnancy ${ }^{(14)}$. Actively proliferating $T$. gondii parasites or tachyzoites transmit to the foetus through vertical transmission in infected pregnant women and can cause congenital toxoplasmosis, which may result in conditions like- retinochorioditis, serious neurological disability, physical deformities and severe tissue destruction (toxoplasmic encephalitis) or even death of the foetus ${ }^{(15)}$. The immune micro-environment at the maternal - foetal interface plays a vital role in embryo implantation and embryonic and placental development ${ }^{(14)}$. T. gondii disrupts the immunity and tolerance balance leading to abnormal pregnancies ${ }^{14)}$.

Any imbalance between oxidative agents and antioxidants can lead to severe problems in pregnant women such as abortion, because pregnancy is characterized by low defence against prooxidizing agents ${ }^{(16)}$. In pregnant women due to $T$. gondii infection free radicals are formed, oxidative stress increases and antioxidizing systems get crippled leading to early pregnancy loss ${ }^{(16)}$. Antioxidants including superoxide dismutase activity, glutathione peroxidase activity, catalase activity, vitamin $\mathrm{C}$, vitamin $\mathrm{E}$, glutathione and albumin concentrations significantly grow high in the serum of the toxoplasmosis aborted women, which is characterized by the free radical released with infection causing increased the oxidative stress ${ }^{(16)}$. Toxoplasmosis was reported to be associated with the stimulation and progress of pregnancy induced-hypertension because of oxidative stress and endothelial dysfunction ${ }^{(17)}$. Increased production of free radicals leads to more lipid peroxidation and then higher oxidative damage in cellular membranes, which includes placental membrane damage due to lipid peroxidase inhibiting the synthesis of prostaglandin 12 enzyme, thus causing this enzyme to malfunction, and then the vessels shrink causing hematopoietic and platelet aggregation ${ }^{(18)}$. This is followed by anaemia, ischemia and increased cell damage. Thus, the occurrence of abnormal placentation and miscarriages is due to an enhanced oxidative process ${ }^{(16)}$. We hypothesize that pregnant 
women are more susceptible to acquire T. gondii infection in Bangladeshi population and oxidative stress may play a pivotal role on the susceptibility and subsequent transmission of T. gondii into developing foetus that can cause congenital toxoplasmosis. Here, in a case-control study among pregnant women and non-pregnant control subjects in the Bangladeshi population, we show that pregnant women are more susceptible to acquire T. gondii infection and have more oxidative stress compared to healthy subjects.

\section{Materials and Methods}

Study subjects and sample collection: This case-control study was carried out on 92 pregnant women and 65 non-pregnant, healthy female volunteers as control. Blood samples from pregnant women were collected from the outpatient department (OPD) of Bangladesh Institute of Research and Rehabilitation in Diabetes, Endocrine and Metabolic Disorders (BIRDEM) Hospital. The female control subjects were enrolled from different areas of Dhaka Metropolitan Area. Anthropometry and baseline characteristics including socio-demographic data, medical history etc. was taken using a pre-set questionnaire. Informed consent was obtained from each study subject. Blood samples from the study subjects were collected in two separate tubes- (i) EDTA containing tube for plasma separation and (ii) lavender cap tube for DNA extraction. After blood collection both the tubes were shaken very gently and kept at $4^{\circ} \mathrm{C}$ for 1 hour. Following incubation, these samples were used for plasma separation and DNA extraction.

Isolation of plasma from whole blood of pregnant women and control subjects: Following blood collection, the EDTA tubes were centrifuged at $1200 \mathrm{~g}$ for 15 minutes in a refrigerated centrifuge machine. Following centrifugation, the supernatant was collected carefully leaving the pellet in the bottom of the tube and transferred into a fresh test tube using a Pasteur pipette. The resulting supernatant is designated as plasma which is maintained at $2-8^{\circ} \mathrm{C}$ while handling. The plasma that was not analysed immediately was apportioned into $0.5-1.0 \mathrm{ml}$ aliquots, stored, and transported at $-20^{\circ} \mathrm{C}$.

Detection of T. gondii specific IgM/IgG in the plasma samples: The seroprevalence of IgG and IgM antibodies against T. gondii was determined by using Toxo IgG/gM rapid test cassette kit (Cortez diagnostics, inc. USA) as per the manufacturer's recommended protocol. Briefly, the kit components, consisting of a strip, a dropper and a buffer solution, was equilibrated with room temperature prior to testing. The strip was taken out of a sealed packet and then one drop of plasma sample and two drops of buffer solution were applied in the S-marked cavity of the kit followed by an incubation for 15 minutes for the bands to form. Band formation regions C, IgG and IgM were designated for control, anti-T. gondii IgG and anti-T. gondii IgM, respectively.

DNA Extraction and Isolation from blood of pregnant women and control subjects: DNA was extracted by phenol-chloroform-isoamyl alcohol methods as previously described ${ }^{(19)}$. Briefly, blood was homogenized by using a rotating blood mixer. Then, $500 \mu \mathrm{l}$ of blood 
was poured into a $1.5 \mathrm{ml}$ microcentrifuge tube and then $950 \mu \mathrm{l}$ of red cell lysis buffer was added to the tube. Afterward the tube was shaken gently until homogenization of the mixture and then spun for $2 \mathrm{~min}$ at $4000 \mathrm{~g}$ in a centrifuge machine ( $\mathrm{Z} 216 \mathrm{M}$, Hermle Labortechnik $\mathrm{GmbH}$, Germany). The supernatant was then discarded and the same step was repeated once to remove haemoglobin. The tube was placed on tissue paper downward for few seconds to get rid of any remaining supernatant but caution was taken so that there was no cross contamination between different samples. Next, $400 \mu \mathrm{l}$ of nucleic lysis buffer was added to the tube and the pellet was resuspended gently by using a micropipette, followed by the addition of $100 \mu \mathrm{l}$ of saturated $5 \mathrm{M} \mathrm{NaCl}$ and $600 \mu \mathrm{l}$ of prechilled phenol-chloroform-isoamyl alcohol to the tube. Following mixing on a rotating blood mixer at room temperature, the tube was then centrifuged for $2 \mathrm{~min}$ at $4000 \mathrm{~g}$. Once the centrifugation was complete, $400 \mu \mathrm{l}$ of supernatant was transferred to a fresh $1.5 \mathrm{ml}$ microcentrifuge tube followed by the addition of $800 \mu \mathrm{l}$ of ice cold $\left(-20^{\circ} \mathrm{C}\right)$ absolute ethanol and shaken gently till DNA appeared as a mucus-like strand in the solution phase. The tube was spun for $1 \mathrm{~min}$ at $6850 \mathrm{~g}$, then the supernatant was discarded carefully and the tube was dried completely in room temperature by placing the tube downward on a tissue paper. Lastly, the pelletcontaining DNA was dissolved in $50 \mu \mathrm{l}$ of Tris-EDTA (TE) buffer. The amount of isolated DNA was estimated using a NanoDrop (NanoDrop1000, US). The normal concentration of the separated DNA was $450 \pm 15 \mathrm{ng} / \mu \mathrm{L}$. This technique generates pure DNA with a normal worth of 260/280 proportion was 1.8 . This was considered as purified DNA solution and kept at $-20^{\circ} \mathrm{C}$ for experimental analysis.

Detection of T. gondii DNA in blood using PCR followed by Agarose Gel Electrophoresis: DNA was isolated from each sample by using the phenol-chloroform-isoamyl alcohol extraction method as described above. T. gondii DNA was detected by conventional PCR targeting 529 bp REP gene with the primers TOXO_F (5'-AGAGACACCGGAA TGCGATCT-3') (MW-6,454.3 $\left.\mathrm{g} / \mathrm{mol}, \mathrm{Tm}=58.2^{\circ} \mathrm{C}\right)$ and TOXO_R $\left(5^{\prime}\right.$-TTCGTCCAAGCCT CCGACT-3') (MW-5,699.7 g/mol, $\left.\mathrm{Tm}=58.7^{\circ} \mathrm{C}\right)$. For each PCR reaction, the forward primer $0.5 \mu \mathrm{l}$, reverse primer $0.5 \mu \mathrm{l}$, Go Taq (Taq polymerase: Promega, Madison, WI USA, www.promega.com ) $12.5 \mu \mathrm{l}, \mathrm{PCR} \mathrm{H}_{2} \mathrm{O} 10.5 \mu \mathrm{l}$ and sample template $1 \mu \mathrm{l}$ (in case of control $1 \mu \mathrm{l} \mathrm{PCR} \mathrm{H}_{2} \mathrm{O}$ was added extra instead of template) were taken in a PCR tube, making the total reaction volume $25 \mu$ l. The PCR program was set for $5 \mathrm{~min}$ at $94^{\circ} \mathrm{C}$ before cycling, followed by 30 cycles of denaturation at $94^{\circ} \mathrm{C}$ for $35 \mathrm{sec}$, annealing at $56^{\circ} \mathrm{C}$ for 1 minute, extension at $72^{\circ} \mathrm{C}$ for $1 \mathrm{~min}$ and finally holding at $4^{\circ} \mathrm{C}$ for $10 \mathrm{~min}$. Amplification was performed with a negative control of $T$. gondii. The amplified DNA was then run through an agarose gel $(2 \%)$ electrophoresis, followed by imaging using a gel doc system (Alphalmager MINI proteinsimple).

Estimation of Total Oxidative Stress (TOS) in plasma: Total oxidative stress was determined as described in literature ${ }^{(20)}$. Briefly, $0.1 \mathrm{ml}$ plasma, $1.9 \mathrm{ml}$ PBS and $1 \mathrm{ml}$ 
acetate buffer were taken in a tube (i.e., plasma was diluted to 20 times). For assay standard, different dilutions of hydrogen peroxide $\left(\mathrm{H}_{2} \mathrm{O}_{2}\right)$ in millimolar concentrations per litre were prepared. Then $25 \mu \mathrm{l}$ of working chromogen solution was then added to the tube, followed by measuring the absorbance at $505 \mathrm{~nm}$ by $6 \mathrm{~min}$ time-scan in UV-VIS spectrophotometer (Shimadzu UV 1800 spectrophotometer). Absorbance of $\mathrm{H}_{2} \mathrm{O}_{2}$ solution with different dilutions at $505 \mathrm{~nm}$ in a six-min time-scan between 6th and 4th minute and the difference between the two absorbance's were calculated to prepare a standard curve. The absorbance values obtained at four to six minutes for every sample against blank were compared with the standard curve to determine TOS in plasma of each study subject.

Estimation of Total Antioxidant Defence (TAD) in Plasma: Total antioxidant defence was measured as described in literature ${ }^{(20)}$. Briefly, $1.0 \mathrm{ml}$ of acetate buffer $(\mathrm{pH}=5.2)$ was taken in a test tube. Twenty-five microliter chromogen reagent (that contains $\mathrm{N}, \mathrm{N}$ dimethyl- p- phenylenediamine sulphate) and $10 \mu \mathrm{FeCl}$ ssolution were added. Ten microliters of 20X diluted plasma was added to the mixture, the antioxidant compounds in the sample reduce the chromogen, quenching the colour and producing a discoloration of the solution, which is proportional to their concentration. For standardization, a standard curve of assay of TAD was prepared by using different dilutions of Trolox (6-hydroxy-2, 5,7,8-tetramethylchroman-2-carboxylic acid), a potent antioxidant and it quenches the colour of the chromogen maximally between $4^{\text {th }}$ and $6^{\text {th }}$ minute. With different concentrations of trolox, a water-soluble form of vitamin $\mathrm{E}$, the difference in absorbance between the absorbance values at $505 \mathrm{~nm}$ at $4^{\text {th }}$ and $6^{\text {th }}$ minute of the 6 minutes' time scan was plotted and a standard curve was constructed and concentration of troloxas a measure of total antioxidant defences was quantified.

\section{Results and Discussion}

T. gondii is ubiquitous in nature and creates public health problems particularly in pregnant women and immunocompromised individuals ${ }^{(5-8,21,22)}$. Therefore, understanding its seroepidemiology and pathogenesis in the high-risk groups particularly in pregnant women is very important for controlling and prevention of Toxoplasma mediated complications for instances abortion, intra-uterine growth restriction, jaundice, hepato-hypertrophy or perhaps intra-uterine death ${ }^{(23)}$.This parasite can also develop neurologic or ocular manifestations like intracranial calcifications, abnormality or retinochoroiditis(23). The main focus of this study is to determine the seroprevalence of $T$. gondiiand its potential modulatory effects on oxidative stress in pregnant women, which may cause congenital toxoplasmosis.

Socio-demographic characteristics of pregnant women subjects: It has been noted in previous studies that there is a relationship between $T$. gondii infection in pregnant women and developing congenital Toxoplasmosis ${ }^{(5)}$.To understand the impact of this 
parasitic infection in pregnant women in Bangladesh, the seroprevalence of $T$. gondii infection was estimated in a group of pregnant women enrolled from Dhaka, Bangladesh. Table 1 demonstrates the socio- demographic characteristics of the pregnant women subjects $(\mathrm{N}=92)$, and the seroprevalence of $T$. gondii infection characterized by the presence of $\operatorname{IgG}$ antibody against $T$. gondii in plasma.

Table 1. Socio-demographic characteristics and seroprevalence of IgG antibody against T. gondii in pregnant women.

\begin{tabular}{lcc}
\hline Parameter & $\begin{array}{c}\text { Cases (Pregnant } \\
\text { women) }\end{array}$ & $\begin{array}{c}\text { Ati-T. gondii IgG } \\
\text { positive }\end{array}$ \\
\hline $\begin{array}{l}\text { Gender No. (\%) } \\
\text { Female }\end{array}$ & $92(100 \%)$ & $23(25 \%)$ \\
Age Sub-groups: No (\%) & & \\
Group-I : 16-25 Years & $23(25 \%)$ & $6(26 \%)$ \\
Group-II : 26-35 Years & $59(64 \%)$ & $16(27 \%)$ \\
Group-III : >36 Years & $10(11 \%)$ & $1(10 \%)$ \\
Stage of Pregnancy: No (\%) & & \\
First Trimester & $21(23 \%)$ & $5(24 \%)$ \\
Second Trimester & $26(28 \%)$ & $2(8 \%)$ \\
Third Trimester & $45(49 \%)$ & $16(36 \%)$ \\
NumberofPregnancy: No (\%) & & \\
First & $24(26 \%)$ & $5(21 \%)$ \\
Second & $33(36 \%)$ & $7(21 \%)$ \\
Third & $25(27 \%)$ & $10(40 \%)$ \\
Fourth & $10(11 \%)$ & $1(10 \%)$ \\
Occupation: No (\%) & & \\
Yes & $25(26 \%)$ & $16(17 \%)$ \\
No & $68(74 \%)$ & $7(8 \%)$ \\
Occupation: No (\%) & & $21(26 \%)$ \\
Housewife & BI (88\%) & $2(18 \%)$ \\
Service & $11(12 \%)$ & \\
\hline
\end{tabular}

Seroprevalence of IgM and IgG antibodies against T. gondii in pregnant women and control subjects:To assess the seroprevalence of anti-T. gondii $\operatorname{IgG}$ and $\operatorname{IgM}$, we used an immunological assay with a rapid test cassette. The presence of IgG and IgM antibodies against $T$. gondii was determined and interpreted as per the manufacturer's guidelines. Out of 92 pregnant women plasma samples, 23 were IgG antibody positive meaning that $25 \%$ of the cases were seropositive for IgG antibodies against T. gondii(Figure 1).Among them, 3 were also positive for both $\operatorname{IgM}$ and $\operatorname{IgG}$ antibodies. Otherhands, among the 65 
samples of the control group only $7(9.2 \%)$ were positive for IgG antibodies against $T$. gondii (Fig. 1). This data primarily indicates that seropositivity of antibodies against $T$. gondiiparasite is more prevalent in pregnant women than non-pregnant control women. Based on the immune assay, it was confirmed that $3 \%$ pregnant women carried acute $T$. gondii infection in their blood indicated by the presence of anti-T. gondii IgM antibody in plasma. However, the increased rate of anti-T. gondii IgG (25\%) in pregnant women confirmed their chronic infection, which is significantly higher than non-pregnant healthy controls $(p=0.013)$. Our data was supported by another study conducted by Margia and colleagues where they have shown that $25.3 \%$ pregnant women who visited Rangpur and Mymensingh Medical colleges possess anti-T. gondii IgG and IgM antibodies in their serum ${ }^{(24)}$. Therefore, we further confirmed that T. gondii infection is moderately present $(25 \%)$ in pregnant women in Dhaka. Our study is different form this study in various aspects for instance (i) we enrolled pregnant women from different areas of Dhaka city and who visited BIRDEM general hospital (ii) for the first time, we investigated oxidative stress and antioxidant defence markers in pregnant women to understand the impact of the parasite on medical complications in pregnancy.

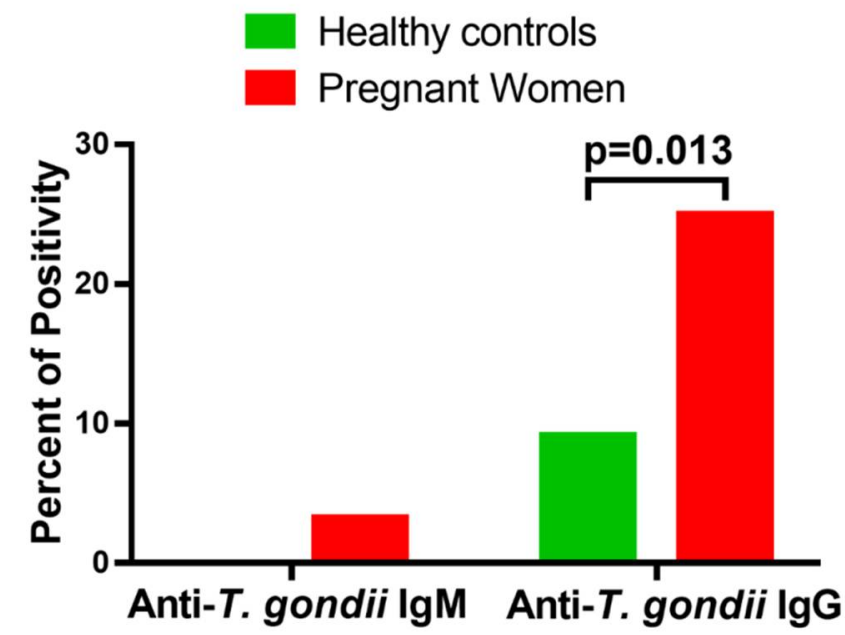

Fig. 1. Seroprevalence of anti- T. gondii IgG and IgM antibodies in pregnant women and non-pregnant control subjects. Percentage comparison of seropositivity of $T$. gondii -specific IgG and IgM antibodies between pregnant women $(\mathrm{N}=92)$ and non-pregnant control $(\mathrm{N}=65)$ subjects. The statistically significant differences between pregnant women and non-pregnant control subjects were calculated by Fisher's exact test. $\mathrm{P}$ value indicated in the figure was considered significant if $\mathrm{p}<0.05$.

PCR assay confirms pregnant women were not acutely infected by T. gondii: To determine whether the $T$. gondii infected pregnant women are chronically or acutely infected, we performed conventional PCR to detect the presence of T. gondii DNA in their blood samples. Although a very small number of pregnant women $(n=3)$ showed both anti-T. 
gondii IgM and IgG positive in plasma, we did not find any parasite specific DNA in their blood using PCR (data not shown), suggesting that the pregnant women were not acutely infected by T. gondii.

Stages of pregnancies of pregnant women and seroprevalence of T. gondii specific IgG antibody: The timeline of pregnancy can be divided into three trimesters each including three months of gestation period. The immune system and the effect of environment of pregnant women are also different along the three trimesters ${ }^{(21)}$. Therefore, it is possible to show differential seropositivity across different trimesters as a result of differential immune response across the trimesters. Hence, in this part, we wanted to see any differences in the seroprevalence of $T$. gondii specific IgG antibody in different trimesters. The absolute number and percent of anti-T. gondii IgG seropositivity are shown in Fig. 2A \& B. It was found that seropositivity of anti-T. gondii IgG was remarkably higher in pregnant women in their third trimester $(35.56 \%)$ compared to pregnant women in their first $(23.81 \%)$ and second trimester $(7.69 \%)$ (Fig. 2B). The association between the stages of pregnancy and the seropositivity of anti-T. gondii IgG antibody was found to be statistically significant ( $\mathrm{p}=0.0107$ by Fisher's exact test) (Fig. 2).
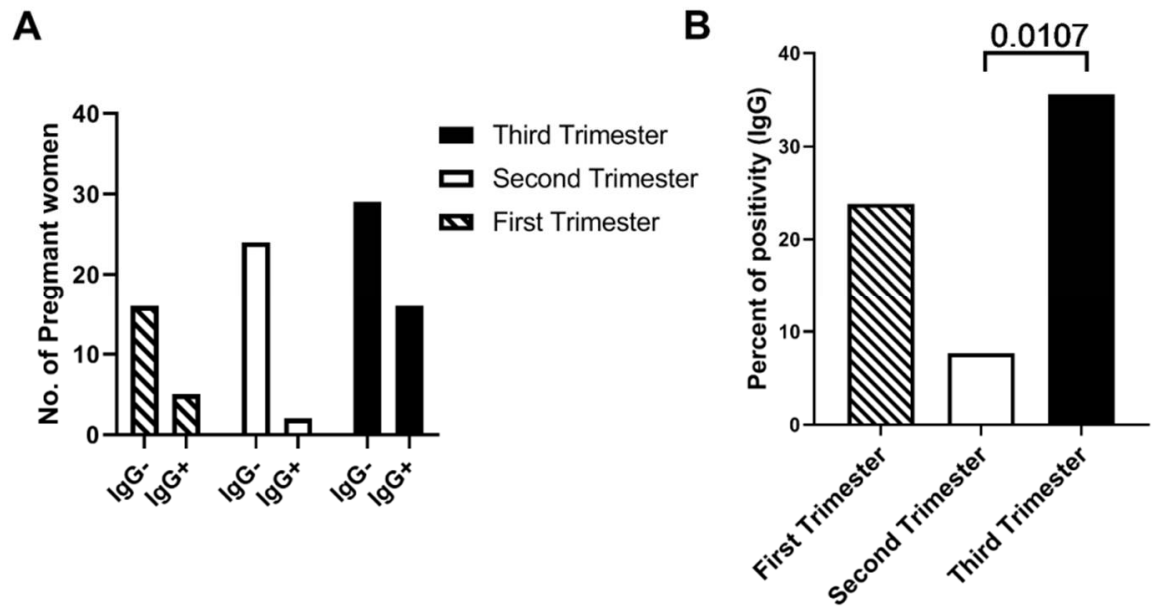

Fig. 2. Seroprevalence of IgG antibody against $T$. gondiiin different stages of pregnancies. Using a rapid test cassette, the seroprevalence of anti- $T$. gondii $\operatorname{IgG}$ antibody was determined and compared between different stages of pregnancies. (A) Comparison between the number of IgG positive and negative pregnant women in different stages of pregnancy. (B) Percentage comparison of IgG positivity in pregnant women in different stages of pregnancies. P-value stated in the figure was calculated by Fisher's exact test and was considered significant if $\mathrm{p}<0.05$.

Contact with cats of pregnant women and seroprevalence of antibodies against T. gondii: Cats play a vital role in the life cycle of $T$. gondii as it is the primary host where the parasite continues its sexual phase of the life cycle. It has been noted by many researchers that cat litter is a potential way of transmission of this parasite and thus elderly and pregnant women are suggested to be kept away from cat and cat litter in to prevent 
infection with the parasite. In accordance with this, we hypothesize that pregnant women who were in contact with cats are more susceptible to acquire $T$. gondii infection than those who did not. To address this, we detected anti-T. gondii IgG antibody in pregnant women with or without cat contact. The absolute number and the percent of anti-T. gondii IgG seropositivity are shown in Fig. 3A \& B. We found that $69.56 \%$ of the $23 \mathrm{~T}$. gondiipositive pregnant women were in contact with cat and there was a significant positive correlation between cat contact and the seroprevalence of anti-T. gondii IgG antibody in pregnant women ( $p<0.0001$ by Fisher's exact test (Fig. 3B). This result can be explained by the fact that cat is the final host of $T$. gondii and contamination with cat faeces is a prominent way of transmission of $T$. gondii oocysts, resulting in acquiring a higher T. gondii infection in pregnant women who were in cat contact. Therefore, pregnant women must avoid any contact with cats, soil, as well as handling and consumption of raw meat and its by-products. Pet cats must only be fed with adequate pet food or cooked food, and they should not be allowed to hunt. Cat sand boxes must be cleaned daily, by people who are not in the high-risk group. The use of gloves for gardening is recommended. As an awareness of the threats of the disease serological follow-up during pregnancy is of great importance in the prevention of congenital toxoplasmosis.

A

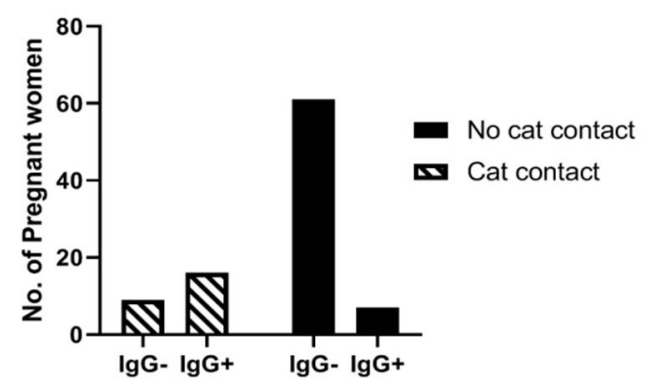

B

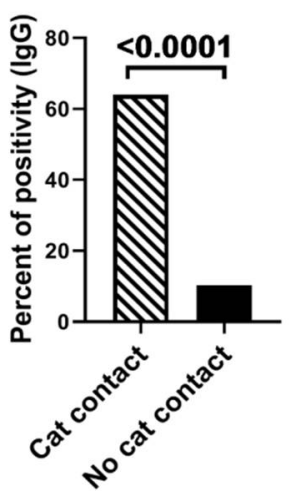

Fig. 3. Seroprevalence of IgG antibody against $T$. gondiiin pregnant women with or without cat contact. Using a rapid test cassette, the seroprevalence of anti-T. gondii IgG antibody was determined and compared between pregnant women with or without cat contact. (A) Comparison between the number of $\operatorname{IgG}$ positive and negative pregnant women who have been in or no contact with cats. (B) Percentage IgG positivity in pregnant women in or no contact with cats. P-values stated in the figure was calculated by Fisher's exact test and considered significant if $\mathrm{p}<0.05$.

Total oxidative stress in plasma of T. gondii infected and non-infected pregnant women: Measurement of oxidative stress in plasma, other body fluids and extracts has been a pivotal focus in bioscience research as oxidative stress may modulate many biological functions associated with host-pathogen interaction, disease pathologies etc. T. gondii 
infection has been noted to be linked to an increase in oxidative stress and causing destruction of cells in toxoplasmosis in certain groups of people ${ }^{(25)}$. Here, we focused to decipher whether the seropositive (detected with $T$. gondii specific IgG antibody) pregnant women would have more oxidative stress than seronegative pregnant women. To assess this, we measured total oxidative stress and total anti-oxidant defence markers between these two groups. It was observed that the concentration of $\mathrm{H}_{2} \mathrm{O}_{2}$, a marker of oxidative stress, was significantly higher in $T$. gondii infected pregnant women than in uninfected pregnant women ( $\mathrm{p}=0.034$ ) (Fig. 4A), suggesting an association between the increase in oxidative stress and T. gondii infection during pregnancy.

A

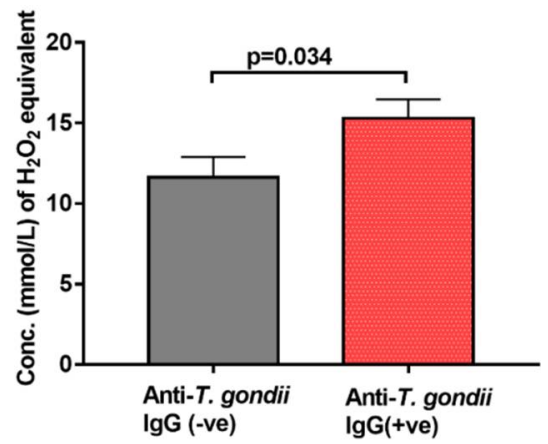

B

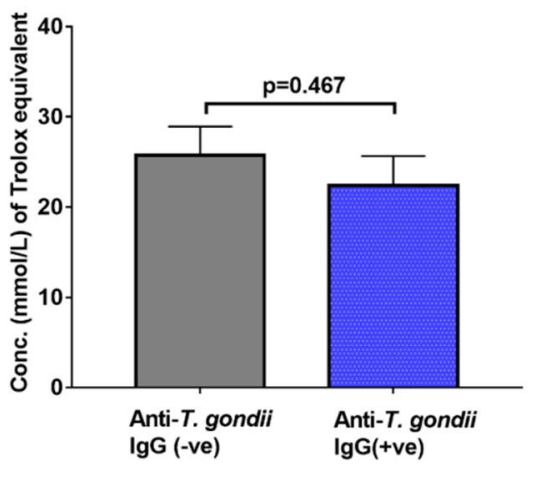

Fig. 4. Total Oxidative Stress (TOS) and Total Antioxidant Defence (TAD) in T. gondii negative and positive pregnant women. (A) TOS was determined by measuring the concentration of $\mathrm{H}_{2} \mathrm{O}_{2}$ equivalent in plasma of anti- T. gondii IgG negative subjects and anti- T. gondii $\mathrm{IgG}$ positive pregnant women. Data are expressed as conc. of $\mathrm{H}_{2} \mathrm{O}_{2}(\mathrm{mmol} / \mathrm{)}) \pm$ Standard Error of Mean (SEM). P-value stated in the figure was calculated by Mann-Whitney test and considered significant if $p<0.05$ (B) TAD was determined by measuring the concentration of Trolox equivalent, a marker of antioxidant defence in the same subjects. Data are expressed as conc. of Trolox $(\mathrm{mmol} /) \pm$ Standard Error of Mean (SEM). P-value stated in the figure was calculated by Mann-Whitney test and considered significant if $p<0.05$.

Later, we also focused to know whether the parasitic infection, along with the increase in oxidative stress, may also reduce antioxidant defence in pregnant women. To address this, we measured the concentration of Trolox (a marker of antioxidant activity) in plasma samples from $T$. gondii infected and uninfected pregnant women. The antioxidant activity was found to be less in T. gondii infected pregnant women compared to T. gondii negative subjects, although it was not statistically significant $(\mathrm{p}=0.467)$ (Fig. 4B). Our data confirm that T. gondii infected pregnant women pose increased total oxidative stress and decreased total antioxidant defences in their plasma. This study was supported by the findings of Mahmood and colleagues where they found pregnant women with Toxoplasmosis had significantly increased levels of malondialdehyde, nitrate and reduced level of antioxidant glutathione indicating oxidative stress ${ }^{(16)}$. This was further supported by Rahman's findings of T. gondii infected pregnant women carry 
decreased activity of catalase and glutathione peroxidase ${ }^{(26)}$. T. gondii infection may have a role on this increased level of oxidative stress in pregnant women. Because, in a recent study, T. gondii was identified as a possible inducer for oxidative stress in male rats ${ }^{(27)}$. Oxidative stress in pregnant women may regulate gene expression at various levels and thus may create many reproductive complications in pregnant women including preeclampsia, miscarriage and preterm labour ${ }^{(28)}$. In addition, increased oxidative stress in pregnant women may also be a factor for congenital transmission of $T$. gondii into developing foetus, which needs to be confirmed in future studies. The study was limited in a number of ways. First, we could not manage to incorporate a suitable positive control in agarose gel electrophoresis of PCR product because of the unavailability of purified DNA from T. gondii. Secondly, for assessing oxidative stress only total oxidative stress and total antioxidant defence markers were studied, however, other specific oxidative stress markers could not be possible to include; however, we plan to address them in the future.

The current study has been designed to find out the seroprevalence of $T$. gondii infection among pregnant women in Dhaka and decipher any possible correlation that exists between T. gondii infections with oxidative stress. This case-control study confirms that T.gondii infection is more prevalent among Bangladeshi pregnant women compared to non-pregnant control group; perhaps pregnant women are more susceptible to acquire T. gondii infection. In addition, we found an increased level of total oxidative stress and decreased level of total antioxidant defence in T. gondii infected pregnant women compared to $T$. gondii negative pregnant women. It is possible that $T$. gondii is responsible for causing oxidative stress, which may be a mediator in immune dysregulation in pregnant women and subsequent adverse conditions in pregnancy. Further studies will be needed to find out the exact mechanism of how $T$. gondii can cause adverse effects in pregnancy and any role of oxidative stress in congenital toxoplasmosis. Since a huge proportion of pregnant women in Bangladesh are likely to be infected by this parasite more research is required to discern the mechanism of how this parasite can bring disastrous consequences to pregnant women and newborns.

\section{Acknowledgements}

The authors thank the Ministry of Education, Bangladesh (Grant No: LS2017560) for funding the project.

\section{Conflict of Interest}

The authors declare no conflicts of interest regarding the publication of this article. 


\section{References}

1. Hill DE, S Chirukandoth and JP Dubey 2005. Biology and epidemiology of Toxoplasma gondii in man and animals.Animal Health Research Reviews. 6(1): 41-62.

2. Dubey JP 2008. The history of Toxoplasma gondii - The first 100 years. J. Eukaryot. Microbiol. 55(6): 467-475.

3. Vogel N, M Kirisits, E Michael, H Bach, M Hostetter, K Boyer, R Simpson, E Holfels , J Hopkins, D Mack,MB Mets,CN Swisher,D Patel ,N Roizen, L Stein, M Stein, S Withers, E Mui, C Egwuagu,J Remington, R Dorfman and R McLeod 1996. Congenital toxoplasmosis transmitted from an immunologically competent mother infected before conception. Clin Infect Dis. 23(5):1055-1060.

4. Havelaar AH, JM Kemmeren and LM Kortbeek 2007. Disease Burden of Congenital Toxoplasmosis. Clin. Infect. Dis. 44(11):1467-1474.

5. Lopes FMR, DD Gonçalves, R Mitsuka-Breganó, RL Freire and IT Navarro 2007. Toxoplasma gondii infection in pregnancy. Brazilian J. Infect. Dis. 11(5): 496-506.

6. Benjamin J, S Luft and Jack 1992. Toxoplasmic Encephalitis in AIDS. Clinical Infectious Diseases.15(2): 211-222.

7. Cong W, GH Liu,QF Meng,W Dong, SY Qin ,FK Zhang, XY Zhang, XY Wang,AD Qian and XQ Zhu 2015. Toxoplasma gondii infection in cancer patients: prevalence, risk factors, genotypes and association with clinical diagnosis. Cancer Lett. 359(2): 307-313.

8. Derouin F and H Pelloux 2008. Prevention of toxoplasmosis in transplant patients. Clin. Microbiol. Infect. 14(12):1089-1101.

9. Jafari Modrek M, R Saravani, M Mousavi, A Salimi Khorashad ans M Piri 2015. Investigation of IgG and IgM Antibodies Against Toxoplasma gondii Among Diabetic Patients. Int. J. Infect. 2(3): 0-4.

10. Ferguson DJP 2009. Identification of faecal transmission of Toxoplasma gondii: Small science, large characters. Int. J. Parasitol. 39(8): 871-875.

11. Dubey JP 2004. Toxoplasmosis - A waterborne zoonosis. Vet. Parasitol. 126(1-2):57-72.

12. Rahman T, A Rahman and S Chakraborty. Infection of Toxoplasma gondii in Humans and Livestock Animals: An Emerging Silent Threat for Bangladesh 2018. Open J. Med. Microbiol. 08(04): 109-117.

13. Naheen CR, S Tarafder, H Sattar and S Khan 2016. Toxoplasma gondii specific IgG avidity assay: Role and implication in the confirmatory diagnosis of acute toxoplasmosis in seropositive pregnant women. J. Bangabandhu Sheikh Mujib Med. Univ. 9(2): 96-99.

14. Zhang D, L Ren, M Zhao, C Yang, X Liu, H Zhang, Y Jiang, X Sun, T Li, L Cui and X Hu 2019. Role of Tim-3 in Decidual Macrophage Functional Polarization During Abnormal Pregnancy With Toxoplasma gondii Infection. Frontiers in immunology. 10 (2): 1550.

15. Buffolano W 2012. Toxoplasmosis in the fetus and newborn. Neonatol A. Pract. Approach to Neonatal Dis. 898-904.

16. Mohammed MA, KI Rajab, KF Al-rawi and MY Al-darwesh 2020. Evaluation Some Antioxidants and Oxidative Stress index in Seropositive Toxoplasmosis in Pregnant Women in Ramadi city of Iraq. 11(12): 701-705. 
17. Al-Kuraishy HM, AH Al-Kuraishi, S Al-Windy and AI Gareeb 2019. Toxoplasmosis and risk of endothelial dysfunction: Role of oxidative stress and pro-inflammatory mediators. Arch. Clin. Infect. Dis. 14(6): 6-11.

18. Smith C and AD Marks 2005. Oxygen Toxicity and Free Radical Injury. Mark's Basic Medical Biochemistry: A Clinical Approach.

19. Iranpur VM and AK Esmailizadeh 2010. Rapid Extraction of High Quality DNA from Whole Blood Stored at $4^{\circ} \mathrm{C}$ for Long Period.

20. Saha P, P Banerjee, L Auddya, P Pal, M Das, M Dutta, S Sen, MC Mondal, A Kumar and UK Biswas 2015. Simple Modified Colorimetric Methods for Assay of Total Oxidative Stress and Antioxidant Defense in Plasma: Study in Diabetic Patients. Archives of Medicine. 7(5): 1

21. Dunn D, M Wallon,F Peyron, E Petersen, C Peckham and R Gilbert 1999. Mother-to-child transmission of toxoplasmosis: risk estimates for clinical counselling. Lancet. 353(9167): 1829-1833.

22. Freppel W, DJP Ferguson,K Shapiro, JP Dubey, PH Puech and A Dumètre 2018. Structure, composition, and roles of the Toxoplasma gondii oocyst and sporocyst walls. Cell Surf. 5:100016

23. Khan K and W Khan 2018. Congenital toxoplasmosis: An overview of the neurological and ocular manifestations. Parasitol. Int. 67(6): 715-721.

24. Margia UH, N Begum, MS Hossain, AR Dey and MZ Alam 2017. Seroprevalence of Toxoplasmosis in Women in Mymensingh and Rangpur in Bangladesh: A Hospital Survey. Bangl. J. Vet. Med. 15(1): 81-85.

25. Dincel GC and HT Atmaca 2016. Role of oxidative stress in the pathophysiology of Toxoplasma gondii infection. Int. J. Immunopathol. Pharmacol. 29(2): 226-240.

26. Abdul-rahman SJ, OI Mahmood and RS Abdul-aziz 2015. Evaluation of Oxidative Stress during Toxoplasmosis in Pregnant Women. International Conference on Chemical, Agricultural and Biological Sciences. 102-106.

27. Hoseiny Asl Nazarlu Z, M Matini, M Bahmanzadeh and F Foroughi-Parvar 2020. Toxoplasma gondii: A possible inducer of oxidative stress in reproductive system of male rats. Iran J. Parasitol. 15(4): 521-529.

28. Duhig K, LC Chappell, AH Shennan 2016. Oxidative stress in pregnancy and reproduction. Obstet. Med. 9(3):113-116. 